\title{
Bolivien: Geduldiges Papier, ferner Staat und NGO-Power
}

Politische Instabilität sowie soziale und wirtschaftliche Probleme haben die Entwicklung der Bibliotheken in Bolivien lange behindert. Noch heute gibt es kein nationales Bibliothekssystem und nur wenig staatliche Unterstützung für Informationseinrichtungen. Zwar wurden in der Vergangenheit unzählige Gesetze und Dekrete verabschiedet, die auch Bibliotheken zum Gegenstand der Regulierung hatten, Behörden schufen, zusammenlegten und auflösten - Auswirkungen auf die Realität der Bibliotheken hatten sie jedoch kaum. Schon 1936 wurde eine Abteilung im Bildungsministerium geschaffen, der alle Bibliotheken des Landes untergeordnet waren, ${ }^{1}$ Spuren von der Aktivität dieses Organs lassen sich aber keine finden. ${ }^{2} 1971$ wurde sogar von der UNESCO ein zehnjähriger „Plan for the Development of Public and School Libraries“ für Bolivien angefertigt. Ein argentinischer UNESCO-Mitarbeiter hatte zuvor die desaströse Lage der öffentlichen Bibliotheken und der Schulbibliotheken in Bolivien dokumentiert. ${ }^{3}$ Er schlug unter anderem die Schaffung einer zentralisierten Struktur des öffentlichen Bibliothekswesens vor. Auch dies wurde jedoch nie umgesetzt. ${ }^{4}$

In der Kolonialzeit waren die Bibliotheken in Bolivien nur den Privilegierten zugänglich. Die bedeutendsten Sammlungen befanden sich in den Konventen, wie der Compañía de Jesús in Potosí. Aus dieser Zeit besteht heute noch die 1606 gegründete Bibliothek der Franziskaner in Tarija. ${ }^{5}$ Außerdem gab es private Bibliotheken. ${ }^{6}$

Die Gründer der ersten öffentlichen Bibliothek in Bolivien, der 1825 ins Leben gerufenen Biblioteca Pública de Chuquisaca in Sucre, waren die von der Aufklärung inspirierten Befreiungskämpfer Antonio José de Sucre und Andrés de Santa Cruz. 1872 wurde diese Bibliothek zur Nationalbibliothek erklärt. Sie ist auch heute noch die bedeutendste Bibliothek in Bolivien. Ihre ersten Buchbestände stammten aus den alten Konventen. Die Fusion mit dem 1883 gegrün-

1 Oporto Ordóñez, L.: Historia de las bibliotecas en Bolivia. o.S.

2 Arze, J.: Aspectos de la Bibliografía Boliviana. S. 125-140, S. 135.

3 Benktson, L. G.: Bolivia: A Plan for the Development of Public and School Libraries. S. 71-75, S. 72 .

4 Lembrecht, C.: Bücher für alle. S. 236-239.

5 Zelaya, T. und M. Salinas: Situación actual de las unidades de información en Bolivia: bibliotecas y centros de documentación. S. 70-85, S. 72.

6 Oporto Ordóñez, L.: Historia de las Bibliotecas en Bolivia. o.S. 
deten Archiv zum Archivo y Biblioteca Nacionales de Bolivia wurde 1935 aus rein finanziellen Gründen vollzogen. ${ }^{7}$ Beide Institutionen beherbergen wichtige Sammlungen, die zum Teil dem Weltdokumentenerbe angehören. ${ }^{8}$ Neben den historischen Büchern aus der Kolonialzeit und einer umfassenden Sammlung von Zeitungen (1825-1907) $)^{9}$ konnte man Anfang des 20. Jahrhunderts verschiedene bedeutende Privatsammlungen inkorporieren, so die Kollektion des Historikers und Bibliographen Gabriel René Moreno (1826-1908) - die vollständigste bibliographische Sammlung bolivianischer Druckwerke von 1634 bis 1908 und die Sammlung von Ernst Otto Rück, die u. a. auch Grammatiken und Wörterbücher in indigenen Sprachen enthält. Sammlungen, die in neuerer Zeit hinzu kamen, waren 2004 die von Alcides Arguedas und 2012 die von Guillermo Lora. ${ }^{10}$

Die Nationalbibliothek war seit ihrer Gründung chronisch unterfinanziert, da die von der Regierung unregelmäßig ausgezahlten Zuwendungen zu gering waren. Käufe waren daher selten, Tausch und Spende waren die Hauptzugangswege. ${ }^{11}$ Nachdem sie traditionell dem Kultusministerium unterstellt war, wurde die Bibliothek 1986 von der bolivianischen Zentralbank übernommen. ${ }^{12} 1995$ gründete diese eine Stiftung, die Fundación Cultural del Banco de Bolivia, um die Belange der ihr unterstellten Kulturinstitutionen besser verwalten zu können..$^{13}$ Angegliedert an Archivo y Biblioteca Nacionales de Bolivia ist seit 2009 auch eine öffentliche Bibliothek, die Biblioteca Pública Gunnar Mendoza Loza. Hier gibt es einen Lesesaal für Kinder, es werden Bücherkisten („Cajas viajeras") in abgelegene Gebiete gesendet und Workshops für die Leseförderung durchgeführt. ${ }^{14}$

Bolivien hat seit dem 19. Jahrhundert verschiedenste Gesetze zur Abgabe von Pflichtexemplaren verabschiedet, in denen die Nationalbibliothek stets als eine der Pflichtabgabebibliotheken genannt war, allerdings wurde die Abgabe

7 Arze Aguirre, R. : La biblioteca nacional de Bolivia y el archivo nacional de Bolivia. S. 141152, S. 141-142.

8 Montalvo Calvimontes, E.: Breve panorama histórico y sistemas actualizados de la catalogación en la biblioteca nacional de Bolivia (con sede en la ciudad de Sucre). S. 1-2.

9 Vernon Jackson, W.: Bolivia, Libraries in. S. 655-659, S. 656.

10 Inch, M.: Gunnar Mendoza en el Archivo y Biblioteca Nacionales de Bolivia. S. 167.

11 Archivo y Biblioteca Nacionales de Bolivia: Archivo y biblioteca nacionales de Bolivia del Banco Central de Bolivia 2008, S. 14-37.

12 Rossells Montalvo, B.: Bibliotecas y educación en Bolivia. S. 12.

13 Inch, M.: Gunnar Mendoza. S. 170-171.

14 Archivo y Biblioteca Nacional de Bolivia: Biblioteca Pública „Gunnar Mendoza Loza“. https://archivoybibliotecanacionalesdebolivia.wordpress.com/2017/02/01/biblioteca-publicagunnar-mendoza-loza-del-abnb/ (8.12.2018) 
von vielen Verlegern und Druckern nicht eingehalten. ${ }^{15} 1968$ wurde das Pflichtexemplarrecht in der Form erneuert, dass die Exemplare nun an eine in La Paz neu eingerichtete Institution, heute Repositorio Nacional genannt, zu senden und dann eines davon (später zwei) an die Nationalbibliothek weiterzuleiten sei. Die dem Kultur- und Tourismusministerium unterstehende Einrichtung in La Paz besitzt allerdings keine adäquaten Räumlichkeiten für die Aufbewahrung der nationalen Schriften und auch eine angemessene bibliothekarische Erfassung bleibt aufgrund des fehlenden Personals aus. Bis heute existiert die Institution mehr auf dem Papier als in der Realität. Die Weiterleitung erfolgt nur lückenhaft, so dass bei Weitem nicht alle in Bolivien veröffentlichten Bücher ihren Weg in die Nationalbibliothek finden. ${ }^{16} 2002$ hat die Nationalbibliothek dennoch die Veröffentlichung der bolivianischen Bibliographie übernommen, ${ }^{17}$ die zuvor, von 1963 an, der privaten Initiative des Verlegers und Buchhändlers Werner Guttentag entsprang. ${ }^{18}$

Neben der Nationalbibliothek ist die bedeutendste öffentliche Bibliothek Boliviens die Biblioteca Mariscal Andrés de Santa Cruz in La Paz, kurz Biblioteca Municipal. Sie wurde 1838 gegründet, nachdem der den Namen verleihende Präsident ein Dekret verabschiedet hatte, das die Gründung von Bibliotheken in allen Hauptstädten der Regionen vorsah. ${ }^{19}$ Die neben den beiden genannten bedeutendsten öffentlichen Bibliotheken befinden sich in Cochabamba (gegründet 1884) und Santa Cruz (gegründet 1997). ${ }^{20}$ Auch in den weiteren neun Distrikten Boliviens gibt es heute Gemeindebibliotheken. ${ }^{21}$ Die öffentlichen Bibliotheken unterstehen seit der ersten Amtszeit von Gonzalo Sánchez de Lozada, als Bolivien durch die „Ley de Participación Popular“ (1994) in 311 Gemeinden („municipios“) aufgeteilt wurde, ${ }^{22}$ den einzelnen Lokalregierungen. ${ }^{23}$ Dies wirkt sich nicht unbedingt positiv auf ihre Entwicklung aus, denn die zuständigen Abteilungen müssen gleichzeitig für soziale Belange wie Gesundheit und Bildung aufkommen und können daher für die Bibliotheken kaum Ressour-

15 Inch, M.: Gunnar Mendoza. S. 173-178.

16 Ebd.

17 Archivo y Biblioteca Nacionales de Bolivia: Historia del Archivo y Biblioteca Nacionales de Bolivia. https://www.archivoybibliotecanacionales.org.bo/index.php/home/historia-abnb (8.12.2018).

18 Vgl. dazu Barnadas, Josep M. (Hrsg.): El libro, espejo de la cultura und Nilges, A.: Nationalbibliographien Lateinamerikas. S. 47-49.

19 Costa de la Torre, A.: El libro en la cultura boliviana de medio siglo (1900-1950). S. 55.

20 Zelaya, T. und M. Salinas: Situación actual. S. 72.

21 Oporto Ordóñez, L.: Bibliotecas en Bolivia. o.S.

22 Pampuch, T. und A. Echalar Ascarrunz: Bolivien. S. 89.

23 Oporto Ordóñez, L.: Bibliotecas en Bolivia. o.S. 
cen bereitstellen. ${ }^{24}$ Je nach Gemeinde, der sie unterstehen, sind die Bibliotheken daher auch völlig unterschiedlich ausgestattet und organisiert. ${ }^{25}$ Insgesamt kann man sagen, dass die Bibliothekslandschaft in den Städten, insbesondere La Paz, Santa Cruz de la Sierra und Cochabamba besser entwickelt ist, während die Etablierung einer ähnlichen Infrastruktur auf dem Lande auf der Strecke geblieben ist. ${ }^{26}$

Um die Entwicklung der öffentlichen und der Schulbibliotheken, insbesondere auf dem Lande, zu unterstützen, wurde 1970 die Institution Banco del Libro („Buchbank“) gegründet. Diese Organisation unterstand dem Kultusministerium und unterhielt noch Anfang des Jahrtausends ein Netz von einigen hundert Bibliotheken. ${ }^{27}$ Mittlerweile wurde diese Institution aufgelöst. ${ }^{28}$ Seit 2012 sammelt nun das Erziehungsministerium im Rahmen der Alphabetisierungskampagne „Bolivia lee“ („Bolivien liest“) gespendete Bücher, um damit Gemeindeund Fahrbibliotheken auszustatten. ${ }^{29}$ Auch über 2.000 neue (kleine) Gemeindebibliotheken wurden im Rahmen der Kampagne begründet. ${ }^{30}$

Ende der 1990er Jahre wurden einige Bibliotheken in Bolivien im Rahmen des staatlichen „Programa para la Investigación Estratégica en Bolivia“ („Programm für die strategische Forschung in Bolivien“) unterstützt, darunter zum ersten Mal auch solche in abgelegenen Regionen wie Pando, Potosí, Tarija oder Beni. Resultat des Programmes war ein Zensus und eine Bestandsaufnahme der Situation der Informationseinrichtungen in Bolivien. In La Paz, wo die öffentliche Bibliothek traditionell mehrere Filialen betreibt, konnte die Fernleihe etabliert werden, einige Bibliotheken erhielten Internetzugang, andere setzten ein

24 Vgl. Saavedra, J.: BiblioWorks: Advocating for the Creation of Libraries for Children in Chuquisaca, Bolivia. S. 25-26 und Rossells, B., L. Oporto und V. Ayllón: Un país desinformado?. S. 21-22.

25 Rossells B.: Bibliotecas y educación. S. 12-18.

26 Rossells, B., L. Oporto und V. Ayllón: Un país desinformado?. S. 268.

27 Vgl. Döllgast, B.: Das Bibliothekswesen in Bolivien an der Schwelle zum Informationszeitalter. S. 278-282, S. 279. sowie Rossells, B., L. Oporto und V.: Un país desinformado?. S. 63-64. 28 Rico, R.: Persönliche Mitteilung

29 Vgl. 500 bibliotecas se equipararon con el programa „Bolivia lee“. In: El Boliviano en vivo (04.12.2014). http://elbolivianoenvivo.com/500-bibliotecas-se-equiparon-con-el-programa-bolivia-lee/ (8.12.2018) und: Otero, P.: Bolivia: celebran campaña en favor de las bibliotecas comunitarias. In: Radio Habana Cuba (2014), (28.6.2016). http://www.la-razon.com/sociedad/Presentan-Bolivia-Lee-pretende-recolectar_0_2705729447.html (8.12.2018).

30 Vgl. Dougherty, D.: Bolivia Sets up Community Libraries Across the Country. https://www. telesurenglish.net/news/-Bolivia-Sets-up-Community-Libraries-Across-the-Country-201412100052.html (14.7.2016) und Tres de cada 100 bolivianos lee dos libros anualmente. In: Página siete - Diario nacional independiente. (09.05.2014).https://www.paginasiete.bo/sociedad/ 2017/5/9/tres-cada-bolivianos-libros-anualmente-137018.html (8.12.2018). 
Ausleihsystem, Datenbanken und Kataloge auf, weitere konnten Lesesäle einrichten oder erstmals einen Freihandbestand einführen. ${ }^{31}$ Diese Einrichtung modernerer Technologien in einigen Bibliotheken in den 1990er Jahren blieb jedoch die Ausnahme. Die Mehrheit der Bibliotheken blieb unterentwickelt. Eine Umfrage unter 281 bolivianischen Informationseinrichtungen zwischen 1996 und 1998 im Rahmen des Programms kam zu dem Schluss, dass das Fehlen eines nationalen Gesetzes oder eines nationalen Bibliothekssystems die Entwicklung vieler Einheiten verhindert. Ihre Situation hänge oft von der institutionellen Zugehörigkeit ab. Am besten ausgestattet seien die großen, traditionellen, öffentlichen Bibliotheken, wie die Nationalbibliothek, die aber Einzelfälle darstellten. ${ }^{32}$

Viele bolivianische Bibliotheken sind als Teil von Regierungsbehörden entstanden. Beispiele sind die Bibliothek des Obersten Gerichts in Sucre, die Biblioteca Económica Financiera des Wirtschafts- und Finanzministeriums in La Paz oder die Kongressbibliothek (Biblioteca y Archivo Histórico de la Asamblea Legislativa Plurinacional), ebenfalls in La Paz und neben der Nationalbibliothek heute eine der wichtigsten Bibliotheken in Bolivien. Sie wurde 1912 eröffnet. $^{33}$ Auch sie war, wie die Nationalbibliothek, lange Zeit auf Spenden angewiesen, da sie über kein offizielles Erwerbungsbudget verfügte, ${ }^{34}$ dank der Regierung von Evo Morales erfährt sie heute mehr Unterstützung. ${ }^{35}$ Sie organisiert ein Kulturprogramm mit Vorträgen, Buchvorstellungen, Kongressen und Seminaren. ${ }^{36}$ Teils betreiben auch die wissenschaftlichen Akademien Bibliotheken, so die nationale Wissenschaftsakademie (Academia Nacional de Ciencias), die Sprachakademie (Academia Boliviana de la Lengua), oder die Gesellschaft der Bolivianischen Geschichte (Sociedad Boliviana de la Historia). ${ }^{37}$

Als bedeutende Spezialbibliothek in Bolivien ist die 1939 gegründete Biblioteca Casto Rojas der Zentralbank in La Paz zu nennen. Die Sammlung an Büchern zu den Themen Wirtschaft und Finanzen wird stetig weiter ausgebaut. ${ }^{38}$ Für den Bereich der ethnologischen und indigenen Themen existieren die in der Universidad Católica Boliviana angesiedelte Biblioteca Etnológica Boliviana, die

31 Ayllón, V. und R. Brinati: Guía para la organización de centros de documentación.

32 Rossells, B., L. Oporto u. V. Ayllón: Un país desinformado?. S. 266-272.

33 Oporto Ordóñez, L.: Historia de las bibliotecas en Bolivia. o.S.

34 Blanco, L.: Diagnóstico del estado de conservación de las colecciones especiales existentes en bibliotecas y archivos de los países del Convenio Andrés Bello. S. 10.

35 Oporto Ordóñez, L.: La Biblioteca del Congreso en su primer centenario.

36 Ebd., S. 15.

37 Oporto Ordóñez, L.: Historia de las bibliotecas en Bolivia. o.S.

38 Bustamante Paco, S.: La Biblioteca „Casto Rojas“ del Banco Central de Bolivia y su aporte a la investigación en el contexto económico y sociocultural de Bolivia. S. 68-71, S. 68-69. 
1981 von dem holländischen Ethnologen Hans van den Berg gegründet wurde, ${ }^{39}$ sowie die Bibliothek des Museo Nacional de Etnografía y Folklore, die größte ethnologische Bibliothek Boliviens. ${ }^{40}$ Eine besonders gute Infrastruktur bieten laut Oporto Oróñez die Bibliotheken im Gesundheitswesen auf. Viele Krankenhäuser hätten gut funktionierende Bibliotheken, zu nennen seien außerdem die Bibliotheken des gastroenterologischen Instituts in La Paz und des Roten Kreuzes. Auch Krankenpflegeschulen und -fakultäten in den Universitäten unterhalten Bibliotheken. ${ }^{41}$

Aufgrund der fehlenden staatlichen Struktur sind viele bolivianische Bibliotheken aus privaten Initiativen entstanden bzw. über Stiftungen ins Leben gerufen worden, wie z. B. im Falle der Bibliotheken der Fundación Pachamama (1995 gegründet). Diese unterhält in Sucre eine der am besten ausgestatteten Bibliotheken in Bolivien. ${ }^{42} \mathrm{Ab}$ Mitte der 1980er Jahre kam es $\mathrm{zu}$ einem regelrechten Boom von Nichtregierungsorganisationen, die oft die Lücken der staatlichen Infrastruktur ausfüllten. Sie gründeten Bibliotheken und Dokumentationszentren und konnten dort häufig, anders als die staatlichen Institutionen, ausgebildetes Personal ein- und modernere Technologien bereitstellen. ${ }^{43}$ Am bekanntesten ist heute das Centro de Documentación e Información de Bolivia (CEDIB). In diesem Informations- und Dokumentationszentrum in Cochabamba kann man sich über soziale Themen informieren, die Bolivien, aber auch das restliche Lateinamerika, betreffen. ${ }^{44}$ Die vom Zentrum bereitgestellte Datenbank memoria histórica („historisches Gedächtnis“) enthält neben bibliographischen Daten auch thematisch erfasste Zeitungsartikel. ${ }^{45}$ Weitere Beispiele sind das Centro de Información y Desarrollo de la Mujer („Informationszentrum und Entwicklung der Frau“), das Centro de Investigación y Documentación para el desarrollo del Beni („Forschungs- und Dokumentationszentrum für die Entwicklung von Beni“) und das Centro de Documentación de la Problemática Indígena („Dokumentationszentrum für die indigene Problematik“), das zu der NGO Apoyo para el Campesino-Indigena del Oriente Boliviano (,Unterstützung für den indigenen Bauern

39 Vgl. dazu van den Berg, H.: Biblioteca Etnológica Boliviana. S. 13.

40 Rossells, B., L. Oporto und V. Ayllón: Un país desinformado? S. 162.

41 Oporto Ordóñez, L.: Bibliotecas en Bolivia. o.S.

42 Speziell zu dieser Bibliothek vgl. Rossells, B., L. Oporto und V. Ayllón: Un país desinformado?. S. 67-68. Der Grundstock von Büchern wurde durch Spendensammlungen von Tür zu Tür in Barcelona gebildet. Die Stiftung bringt die Bücher auch in bolivianische Gefängnisse und in Randgebiete und wird von der Regierung Kataloniens unterstützt.

43 Ebd., S. 26-28.

44 Biblioteca CEDIB. http://cedib.org/biblioteca/ (8.12.2018). Vgl. auch Castro Aliaga, C.: Library Services for Latin American Indiginous Populations. S. 135.

45 Castro Aliaga, C.: Library Services for Latin American Indiginous Populations. S. 135. 
im Osten Boliviens“) gehört. Einige Entwicklungsorganisationen und ausländische Institutionen unterhalten heute in Bolivien Bibliotheken, die besser ausgestattet sind als die landeseigenen. Ein Beispiel ist das Goethe-Institut in La Paz. ${ }^{46}$ Sehr aktiv im Bereich der Bibliotheken in der Region Chuquisaca ist auch die 2005 gegründete US-amerikanische NGO Biblioworks. Sie hilft den armen, ländlichen Gemeinden und Schulen bei der Gründung neuer Bibliotheken. Bisher hat Biblioworks 12 kleine Bibliotheken und vier „Leseecken“ gegründet. ${ }^{47}$ Ähnlich arbeitet die französische Organisation de Solidarité International, die Bibliotheken in Sucre und Santa Cruz de la Sierra aufgebaut hat, um die vom Bildungssystem ausgeschlossene Bevölkerung mit Büchern zu versorgen. ${ }^{48}$

1984 wurde in Santa Cruz eine öffentliche Kinder- und Jugendbibliothek eröffnet, die erste dieser Art in Bolivien. ${ }^{49}$ In diesem Bereich stechen aber auch zwei weitere, aus internationalen Spenden finanzierte Initiativen heraus: die Biblioteca Kiskhaluru der Bauerngemeinde in Tomina in der Nähe von Sucre und die Biblioteca T'huruchapitas in Cochabamba. ${ }^{50}$

Die wichtigste Universitätsbibliothek Boliviens befindet sich in La Paz an der Universidad Mayor de San Andrés (UMSA). Sie besitzt bedeutende Altbestände (u. a. Handschriften aus dem 16. Jahrhundert). ${ }^{51}$ Teils wurden private Sammlungen aufgekauft. ${ }^{52}$ Neben der Bibliothek der UMSA sind die Zentral- und Institutsbibliotheken der Universidad Mayor de San Simón (UMSS) in Cochabamba, der Universidad Mayor San Francisco Xavier in Sucre (sie erbte Bestände der Compañía de Jesús und beherbergt heute einen bedeutenden Bestand an theologischen, juristischen und philosophischen Werken ${ }^{53}$, der Universidad Gabriel René Moreno in Santa Cruz (hier sticht besonders die Bibliothek der technologischen Fakultät heraus ${ }^{54}$ ) und der Universidad Católica Boliviana in La Paz bedeutend. Letztere konnte 2006 in ein neues Gebäude ziehen - mit 200 Leseplätzen auf sechs Stockwerken $;^{55}$ sie ist außerdem die einzige Bibliothek in Boli-

46 Oporto Ordóñez, L.: Bibliotecas en Bolivia, o.S.

47 Saavedra, J.: BiblioWorks. S. 13-15; S. 26. Vgl. auch Biblioworks - Volunteer through the GivingWay free network. https://www.givingway.com/organization/biblioworks (8.12.2018).

48 Bolivia: Bus biblioteca para Cochabamba (2011-2013). http://www.solidarite.asso.fr/BOLIVIA-Un-Bus-biblioteca-para, (29.6.2016).

49 Rossells M, B.: Bibliotecas y educación. S. 18.

50 Oporto Ordóñez, L.: Historia de las bibliotecas en Bolivia. o.S.

51 Blanco, L.: Diagnóstico del estado. S. 12.

52 Oporto Ordóñez, L.: Historia de las bibliotecas en Bolivia. o.S.

53 Ebd.

54 Sistema Bibliotecario - Facultad de Tecnología. http://biblio.fcet.uagrm.edu.bo/index.aspx?AspxAutoDetectCookieSupport=1, (8.12.2018).

55 http://www.ucb.edu.bo/biblioteca/ (8.12.2018). 
vien, die einen Fernleihservice anbietet. ${ }^{56}$ Die einzelnen Bibliotheken der Fakultäten und Institute arbeiteten lange Zeit unabhängig voneinander, eine gewisse Zentralisierung setzte sich erst in den 1990er Jahren durch. 1999 gab es erstmals ein Treffen aller Universitätsbibliotheken in La Paz, bei dem die Gründung des nationalen Systems der Universitätsbibliotheken (Sistema Nacional de Bibliotecas Universitarias) beschlossen wurde. ${ }^{57}$ Wichtigstes Thema der seither stattfindenden Treffen ist stets die Etablierung von Online-Katalogen gewesen..$^{58}$ Allerdings mangelte es noch lange an der computertechnischen Grundausstattung für diese Pläne. ${ }^{59}$ Heute hat jede der genannten Universitäten einen Online-Katalog, der alle Teilbibliotheken der jeweiligen Universität umfasst.

Einen nationalen Bibliotheksverband gibt es in Bolivien nicht. ${ }^{60}$ Auch eine nationale Fernleihe wurde nie etabliert. ${ }^{61}$ Aufgrund des Fehlens eines landesweiten Netzwerks öffentlicher Bibliotheken erfolgt die Entwicklung und Planung des bolivianischen Bibliothekswesens auf lokaler Ebene. Einige Bibliotheken haben sich in Eigeninitiative in Netzwerken zusammengeschlossen. So gibt es zum Beispiel das Netzwerk der Informationseinrichtungen für Ethnologie (Red de Información Etnológica Boliviana - Redetbo ${ }^{62}$ ) oder den Zusammenschluss der öffentlichen Bibliotheken in El Alto (Red Municipal de Bibliotecas de El Alto - REBIMA). ${ }^{63}$ Die Fundación Patiño unterhält ebenfalls ein Netzwerk von Bibliotheken (öffentliche und Schulbibliotheken) in Cochabamba (u. a. die Bibliothek des Centro Pedagógico y Cultural Simón I. Patiño) und widmet sich in dieser Region der Leseförderung. ${ }^{64}$

Die Knappheit bzw. das Fehlen von festen Budgets für den Bucherwerb ist ein grundlegendes Problem bolivianischer Bibliotheken. ${ }^{65}$ Aufgrund dessen

56 Rico, R.: Persönliche Mitteilung.

57 Rossells, B., L. Oporto und V. Ayllón: Un país desinformado?. S. 54-55.

58 Vgl. z. B. Comité Ejecutivo de la Universidad Boliviana, Secretaría Nacional de Investigación, Ciencia y Tecnología u. Universidad Técnica del Beni (Hrsg.): III Reunión Nacional de Bibliotecas Universitarias. S. 3.

59 Ebd., S. 7-8.

60 IFLA: IFLA World Reports, 2010. https://db.ifla-world-report.org/home/map (8.12.2018).

61 Rico, R.: Persönliche Mitteilung.

62 REDETBO. http://cepaoruro.org/?option=com_content\&view=article\&id=392:redetbo\&catid=24:redetbo\&Itemid=7 (8.12.2018).

63 Zapana Calderón, H. und S. Monasterios Calzada: Sistema de gestión de la información para la Red Municipal de Bibliotecas de El Alto. S. 21-26.

64 Fundación Simón I. Patiño: Unidades de Información. http://centropatino.fundacionpatino.org/areas/unidades-de-informacion/ (8.12.2018). Vgl. auch Fundación Simón I. Patiño: Lectura y desarrollo : una experiencia original en Bolivia. S. 20.

65 Rossells, B., L. Oporto u. V. Ayllón: Un país desinformado?. S. 30. 
sind die meisten auf den Zugang über Spenden und Tausch angewiesen. ${ }^{66}$ Eine Umfrage unter 41 Bibliotheken zwischen 2003 und 2004 ergab, dass der Bucherwerb deshalb nicht strategisch, sondern nur sporadisch stattfindet. Nur $66 \%$ der befragten Einrichtungen hatten überhaupt ein festes Budget zur Verfügung. ${ }^{67}$

Ein großer Teil des Personals ist in den Bibliotheken Boliviens nicht ausgebildet. So kam eine Studie von 2005 zu dem Schluss, dass nur etwa die Hälfte der beschäftigten Bibliothekare in Bolivien dies auch von Beruf sind. ${ }^{68}$ Die bibliothekarische Ausbildung erfolgt in Bolivien über den Studiengang Bibliotecología y Ciencias de la Información der Universidad Mayor de San Andrés an der Facultad de Humanidades y Ciencias de la Educación. ${ }^{69}$ Es können die Abschlüsse „Licenciatura“ (5 Jahre: Staatsexamen), „Bachiller Superior“ (4 Jahre: Bachelor) oder „Técnico Superior“ (3 Jahre, etwa: fachgeprüfter Bibliothekar) erworben werden. ${ }^{70}$ Der Studiengang wurde Anfang der 1970er Jahre etabliert. Vorher gab es in Bolivien keine bibliothekarische Ausbildung. ${ }^{71}$

Ab 1959 wurden in ganz Lateinamerika bibliothekarische Berufsorganisationen gegründet, was auch in Bolivien zur Geburt der Asociación Boliviana de Bibliotecarios führte, die allerdings 1974 reorganisiert werden musste ${ }^{72}$ und dann von der Bildfläche verschwand. Im Jahr 2000 fand auf Initiative des Goethe-Instituts in La Paz erstmals eine internationale Konferenz zum Bibliothekswesen statt. Eines der Ziele war die Neu-Gründung eines nationalen Bibliotheksverbandes. ${ }^{73}$ Daraufhin wurde 2002 offiziell das Colegio de Profesionales en Ciencias de la Información (CPCIB) ins Leben gerufen, heute der nationale Berufsverband in Bolivien. ${ }^{74}$ Das CPCIB kooperiert mit anderen in der Bibliothekswelt aktiven Organisationen wie der Fundación Simón I Patiño oder dem „Programa

66 Ayllón, V. und R. Brinati: Guía S. 13-14; S. 20.

67 Rossells, B., L. Oporto und V. Ayllón: Un país desinformado?. S. 295.

68 López Z., E. und Torrico, E., Baldivia R., A.: Dinámica económica de la cultura en Bolivia. S. 103.

69 Bibliotecología y Ciencias de la Información. Universidad Mayor de San Andrés. http:// www.universia.com.bo/estudios/universidad-mayor-san-andres/bibliotecologia-ciencias-informacion/st/177531 (8.12.2018)

70 Universidad Mayor de San Andrés: Bibliotecología: Información. http://bibliotecologia.umsa.bo/oferta-academica (8.12.2018). Vgl. auch Múnera-Torres, M.: Perfiles de formación bibliotecológica en algunas universidades de América del Sur. S. 95-100; S. 97-98.

71 Arteaga-Fernández, F.: Historia de la carrera de bibliotecología.

72 Oporto Ordóñez, L.: Historia de las bibliotecas en Bolivia. o.S.

73 Döllgast, B.: Das Bibliothekswesen in Bolivien. S. 278.

74 Morales Bellido, H.: El Colegio de Profesionales en Ciencias de la Información de Bolivia CPCIB. Cinco años de labor (2005-2010). S. 45-53, S. 46. Vgl. auch: Colegio de Profesionales en Ciencia de la Información de Bolivia. http://cpcib.blogspot.com/ (8.12.2018). 
de Investigación estratégica en Bolivia“", ${ }^{75}$ organisiert Kurse, Vorträge und Fortbildungen zum Thema Bibliotheken, ${ }^{76}$ und vergibt seit 2008 jährlich einen Preis („Premio Mariscal Andrés de Santa Cruz“) an Institutionen, die im Bereich der Informations- und Bibliothekswissenschaft oder der Informationsinfrastruktur besonders herausragen. ${ }^{77}$ Es gibt verschiedene lokale und regionale Verbände, die teils vom CPCIB unterstützt werden. ${ }^{78}$ Hervorzuheben ist die lokale Asociación de bibliotecarios de Oruro, die schon seit über 40 Jahren besteht und zeitweise der einzige bibliothekarische Berufsverband in Bolivien war. ${ }^{79}$

Die Bücher stehen in den bolivianischen Bibliotheken meist in geschlossenen Magazinen, Freihandbereiche gibt es nur wenige und auch die Ausleihe nach Hause ist nicht überall üblich. ${ }^{80}$ Zur Klassifikation und Aufstellung der Bestände wird häufig die DDC verwendet, so auch in der Nationalbibliothek ${ }^{81}$ oder in der Kongressbibliothek. ${ }^{82}$ Die Katalogisierung erfolgt meist nach den Angloamerikanischen Katalogisierungsregeln. ${ }^{83}$ Derzeit wird in der Nationalbibliothek der Übergang zu RDA vorbereitet. ${ }^{84}$ Wegen Mangel an Personal bleiben die Bestände in vielen Bibliotheken jedoch teils auch unkatalogisiert. ${ }^{85}$ Bis heute wird nicht im Verbund katalogisiert ${ }^{86}$ und vielen Einrichtungen fehlt es an technischer Ausstattung für die elektronische Erfassung der Daten. ${ }^{87}$ In der Nationalbibliothek wurde Mitte der 1990er Jahre mit der Digitalisierung der Katalogdaten begonnen, mithilfe einer von der UNESCO entwickelten DatenbankSoftware. ${ }^{88}$ Viele Bibliotheken in Bolivien benutzen dieses kostenlose Programm seit den späten 1980er Jahren (Microisis, oder später CDS/ISIS) ${ }^{89}$

75 Morales Bellido, H.: El Colegio de Profesionales. S. 47.

76 Ebd., S. 48-49.

77 Ebd., S. 50.

78 Ebd., S. 51.

79 Fernández de Zamora, R.: Directorio de asociaciones de bibliotecarios y profesiones afines de América Latina y el Caribe.

80 Payer, M.: Zum Informationswesen Boliviens.

81 Arze Aguirre, R.: La biblioteca nacional. S. 151.

82 Oporto Ordóñez, L.: La Biblioteca del Congreso. S. 15.

83 Zelaya, T. und.. M. Salinas: Situación actual. S. 76.

84 El ABNB destaca la labor del bibliotecario boliviano.

85 Poco desarrollo de Bibliotecas Virtuales en América Latina.

86 Pacheco Balanza, M.: Persönliche Mitteilung.

87 Rossells, B., L. Oporto und V. Ayllón: Un país desinformado?. S. 271.

88 Montalvo Calvimontes, E.: Breve panorama histórico. S. 4. Vgl. auch Arze Aguirre, R.: Biblioteca Nacional de Bolivia. S. 35.

89 Ayllón, V. und R. Brinati: Guía. S. 50-51. CDS/ISIS ist zwar kein Bibliothekssystem, aber Bibliothekssysteme können darauf basieren. Vorteil der Software ist, dass sie relativ leicht angewendet werden kann ohne IT-Kenntnisse zu besitzen. Vgl. Smet, E.: The CDS/ISIS software as an omnipresent tool for Latin American libraries and documentation centres. S. 4-5. 
Auch wenn die Daten teils digital erfasst sind, besitzen bis heute nur wenige bolivianische Bibliotheken einen Online-Katalog. Die Universitätsbibliotheken bieten hier ein positives Beispiel. Der Katalog der Nationalbibliothek befindet sich derzeit im Aufbau. ${ }^{90}$ Webseiten und Kataloge im Netz funktionieren allerdings oft nur schwerfällig.

Die Digitalisierung in Bolivien steckt noch in den Kinderschuhen. Die Nationalbibliothek ist seit 2015 mit 101 Dokumenten in der von der Asociación de Estados Iberoamericanos para el Desarrollo de las Bibliotecas Nacionales de Iberoamérica („Vereinigung der Iberoamerikanischen Staaten für die Entwicklung der Nationalbibliotheken Iberoamerikas“, ABINIA) initiierten und von der spanischen Nationalbibliothek aus koordinierten Biblioteca Digital del Patrimonio Iberoamericano („Digitale Bibliothek des iberoamerikanischen Kulturerbes“) vertreten. ${ }^{91}$ Im Februar 2017 kündigte sie an, noch im selben Jahr ein Digitalisierungszentrum einzuweihen, um den bereits begonnenen Prozess der Digitalisierung weiterzuführen..$^{92}$ Seit 2013 arbeitet auch die Gemeindebibliothek in Cochabamba an der Digitalisierung ihrer Bestände, die insbesondere als Maßnahme zur Bestandserhaltung gesehen wird. Dafür wurde ein Budget des Kulturbeauftragten der Stadt reserviert. ${ }^{93}$ Einige Universitätsbibliotheken besitzen Repositorien, in denen Lehrende ihre wissenschaftlichen Arbeiten veröffentlichen. Im Bereich virtueller Fachbibliotheken ist die 2005 frei geschaltete Biblioteca Virtual de Salud Pública (,Virtuelle Bibliothek der öffentlichen Gesundheit“) zu nennen, die nicht nur eine bibliographische Meta-Suche über die Kataloge der teilnehmenden Einrichtungen ermöglicht, sondern auch den Zugang zu Zeitschriften zum Thema (darunter Open-Access-Zeitschriften) bietet, sowie einen Webseiten-Guide wichtiger Institutionen. ${ }^{94}$ Die Koordination des Projektes unterlag dem bolivianischen Ministerium für Gesundheit und Sport, unterstützt wurde es von der panamerikanischen Gesundheitsorganisation (Organización Panamericana de la Salud). Beigetragen haben insgesamt 44 Institutionen, dar-

90 Archivo y Biblioteca Nacional de Bolivia. https://www.archivoybibliotecanacionales.org. bo/ (8.12.2018).

91 Archivo y Bibliotecas Nacionales de Bolivia: ABINIA-ABNB Notas sobre el X-EIC. https:// archivoybibliotecanacionalesdebolivia.wordpress.com/abinia-abnb-notas-sobre-el-x-eic/ (8.12.2018).

92 Archivo y Biblioteca Nacional de Bolivia: El ABNB entra de lleno a la era de ladigitalización, 2017. https://archivoybibliotecanacionalesdebolivia.wordpress.com/2017/02/07/el-abnb-entrade-lleno-a-la-era-de-la-digitalizacion/ (8.12.2018).

93 Departamento de Patrimonio y Servicios Culturales: Biblioteca Municipal de Cochabamba será digitalizada. https://culturacbba.wordpress.com/2013/04/23/biblioteca-municipal-de-cochabamba-sera-digitalizada/ (8.12.2018).

94 Biblioteca Virtual de Salud Pública. http://saludpublica.bvsp.org.bo/php/index.php (8.12.2018). 
unter Ministerien, Krankenhäuser, Schulen, NGOs, Universitäten und internationale Organisationen. ${ }^{95}$

Was den Aufbau eines nationalen Bibliothekssystems in Bolivien und den Ausbau staatlicher Unterstützung für Informationseinrichtungen angeht, gab es 2013 einen Hoffnungsschimmer, denn in diesem Jahr wurde unter der Präsidentschaft von Evo Morales ein Buch- und Bibliotheksgesetz („Ley del Libro y la Lectura ,Oscar Alfaro“") verabschiedet, das die Schaffung eines nationalen Bibliotekssystems (Sistema Plurinacional de Archivos y Bibliotecas) unter Leitung des Bildungs- und des Kulturministeriums beinhaltet. Beide Ministerien werden hier als verantwortlich dafür benannt, einen nationalen Plan für die Buchproduktion und die Leseförderung („Plan Plurinacional de Fomento al Libro y la Lectura“) zu implementieren. Jede Regierungsebene soll laut dieses Gesetzes einen solchen Plan verabschieden und neue öffentliche Bibliotheken sollen gegründet werden. ${ }^{96}$ Bisher hat das Gesetz allerdings, ähnlich wie alle vorherigen, keine nennenswerte Wirkung gezeigt.

\section{Literaturverzeichnis}

500 bibliotecas se equipararon con el programa „Bolivia lee“. In: El Boliviano en vivo (04.12.2014). http://elbolivianoenvivo.com/500-bibliotecas-se-equiparon-con-elprograma-bolivia-lee/ (8.12.2018).

Archivo y Biblioteca Nacional de Bolivia. https://www.archivoybibliotecanacionales.org.bo/ (8.12.2018).

Archivo y Biblioteca Nacional de Bolivia: Biblioteca Pública „Gunnar Mendoza Loza“ . https:// archivoybibliotecanacionalesdebolivia.wordpress.com/2017/02/01/biblioteca-publicagunnar-mendoza-loza-del-abnb/ (8.12.2018).

Archivo y Biblioteca Nacional de Bolivia: El ABNB entra de lleno a la era de ladigitalización, 2017. https://archivoybibliotecanacionalesdebolivia.wordpress.com/2017/02/07/elabnb-entra-de-lleno-a-la-era-de-la-digitalizacion/ (8.12.2018).

Archivo y Biblioteca Nacionales de Bolivia: Historia del Archivo y Biblioteca Nacionales de Bolivia. https://www.archivoybibliotecanacionales.org.bo/index.php/home/historiaabnb, (8.12.2018).

Archivo y Biblioteca Nacionales de Bolivia: Archivo y biblioteca nacionales de Bolivia: una historia en común 1825-1943. Sucre: Fundación Cultural del Banco Central de Bolivia 2008.

95 Oro, N.: Biblioteca Virtual en Salud Pública (bvsp) y temáticas Bolivia.

96 Saavedra, J.: BiblioWorks. S. 11-12. Vgl. auch: Asamblea Legislativa Plurinacional: Ley del libro y la lectura „Oscar Alfaro“ 2013 und Oporto Ordóñez, L.: Historia de las bibliotecas en Bolivia. o.S. 
Archivo y Bibliotecas Nacionales de Bolivia: ABINIA-ABNB Notas sobre el X-EIC. https://archivoybibliotecanacionalesdebolivia.wordpress.com/abinia-abnb-notas-sobre-el-x-eic/ (8.12.2018).

Arteaga-Fernández, Fernando: Historia de la carrera de bibliotecología. In: Revista de Bibliotecología y Ciencias de la Información 8 (2003) H. 13, (4.7.2016). S. 151-156.

Arze, José Roberto: Aspectos de la Bibliografía Boliviana. In: El libro, espejo de la cultura ; estudios sobre la cultura del libro en Bolivia ; dedicados a Werner Guttentag en su septuagesimo aniversario. Hrsg. von Josep M. Barnadas. Cochabamba u. a.: Los Amigos del Libro 1990. S. 125-140.

Arze Aguirre, René: La biblioteca nacional de Bolivia y el archivo nacional de Bolivia. In: Anuario/Sucre (1994/95). S. 141-152.

Arze Aguirre, René: Biblioteca Nacional de Bolivia. In: Historia de las bibliotecas nacionales de Iberoamérica: pasado y presente. Hrsg. von José Moreno de Alba u. Ramírez Leyva, y Elsa M. México: UNAM; Instituto de Investigaciones Bibliográficas, Centro Universitario de Investigaciones Bibliotecológicas 1995. S. 35.

Asamblea Legislativa Plurinacional: Ley del libro y la lectura „Oscar Alfaro“ 2013. http://www. wipo.int/edocs/lexdocs/laws/es/bo/bo060es.pdf, (22.02.2018)

Ayllón, Virginia u. Rossana Brinati: Guía para la organización de centros de documentación. La Paz: Fundación PIEB 2001.

Barnadas, Josep M. (Hrsg.): El libro, espejo de la cultura ; estudios sobre la cultura del libro en Bolivia ; dedicados a Werner Guttentag en su septuagesimo aniversario. Cochabamba u. a.: Los Amigos del Libro 1990.

Benktson, L. G.: Bolivia: A Plan for the Development of Public and School Libraries. In: International Library Review 10 (1978) H. 1. S. 71-75.

Biblioteca CEDIB. http://cedib.org/biblioteca/, (8.12.2018).

Biblioteca Central Universidad Católica Boliviana „San Pablo“. http://www.ucb.edu.bo/biblioteca/ (8.12.2018).

Biblioteca Virtual de Salud Pública. http://saludpublica.bvsp.org.bo/php/index.php (8.12.2018).

Bibliotecología y Ciencias de la Información. Universidad Mayor de San Andrés. http://www. universia.com.bo/estudios/universidad-mayor-san-andres/bibliotecologia-ciencias-informacion/st/177531 (8.12.2018).

Biblioworks - Volunteer through the GivingWay free network. https://www.givingway.com/organization/biblioworks (8.12.2018).

Blanco, Lourdes: Diagnóstico del estado de conservación de las colecciones especiales existentes en bibliotecas y archivos de los países del Convenio Andrés Bello. http://unesdoc. unesco.org/images/0009/000943/094302S0.pdf, (8.12.2018).

Bolivia: Bus biblioteca para Cochabamba (2011-2013). http://www.solidarite.asso.fr/BOLIVIAUn-Bus-biblioteca-para, (29.6.2016).

Bustamante Paco, Sikorina: La Biblioteca „Casto Rojas“ del Banco Central de Bolivia y su aporte a la investigación en el contexto económico y sociocultural de Bolivia. In: Fuentes, Revista de la Biblioteca y Archivo Histórico de la Asamblea Legislativa Plurinacional 9 (2015) H. 37. S. 68-71. http://www.revistasbolivianas.org.bo/scielo.php?script=sci_arttext\&pid=S1997-44852015000200009\&lng=es\&nrm=iso (8.12.2018).

Campaña 'Bolivia Lee' 2017 pretende recolectar más de 210.000 libros. In: La Razón (8.5.2017). http://www.la-razon.com/sociedad/Presentan-Bolivia-Lee-pretende-recolectar_0_2705729447.html (8.12.2018).[ 
Colegio de Profesionales en Ciencia de la Información de Bolivia. http://cpcib.blogspot.com/ (8.12.2018).

Comité Ejecutivo de la Universidad Boliviana, Secretaría Nacional de Investigación, Ciencia y Tecnología u. Universidad Técnica del Beni (Hrsg.): III Reunión Nacional de Bibliotecas Universitarias. Trinidad 6,7, y 8 de Diciembre de 2001. Bolivia: Comité Ejecutivo de la Universidad Boliviana 2002.

Costa de la Torre, Arturo: El libro en la cultura boliviana de medio siglo (1900-1950). La Paz: Difusión 1972.

Departamento de Patrimonio y Servicios Culturales: Biblioteca Municipal de Cochabamba será digitalizada. https://culturacbba.wordpress.com/2013/04/23/biblioteca-municipal-decochabamba-sera-digitalizada/ (8.12.2018).

Döllgast, Brigitte: Das Bibliothekswesen in Bolivien an der Schwelle zum Informationszeitalter. In: Bibliotheksdienst 35 (2001) H. 3. S. 278-282. https://www.degruyter.com/view/j/ bd.2001.35.issue-3/bd.2001.35.3.278/bd.2001.35.3.278.xml (8.12.2018).

Dougherty, David: Bolivia Sets up Community Libraries Across the Country. https://www.telesurenglish.net/news/-Bolivia-Sets-up-Community-Libraries-Across-the-Country-201412100052.html (8.12.2018).

El ABNB destaca la labor del bibliotecario boliviano. In: Correo del sur (30.6.2016). http://correodelsur.com/cultura/20160630_el-abnb-destaca-la-labor-del-bibliotecario-boliviano. html (8.12.2018) [

Fernández de Zamora, Rosa María: Directorio de asociaciones de bibliotecarios y profesiones afines de América Latina y el Caribe. La Haya: Ifla Headquarters 1998 (IFLA professional reports 55).

Fundación Simón I. Patiño: Unidades de Información. http://centropatino.fundacionpatino. org/areas/unidades-de-informacion/ (8.12.2018).

Fundación Simón I. Patiño: Lectura y desarrollo : una experiencia original en Bolivia. Genf: Fundación Simón I. Patiño 1996.

IFLA: IFLA World Reports, 2010. https://db.ifla-world-report.org/home/map (8.12.2018).

Inch, Marcela: Gunnar Mendoza en el Archivo y Biblioteca Nacionales de Bolivia. Notas para su estudio. In: Ciencia y Cultura 33 (2014) 165-181, (27.6.2016).

Lembrecht, Christina: Bücher für alle: Die UNESCO und die weltweite Förderung des Buches 1946-1982. Berlin, Boston, Mass.: Walter de Gruyter 2013.

López Z., Eduardo u. Torrico, Erick, Baldivia R., Alejandra: Dinámica económica de la cultura en Bolivia. Bogotá: Convenio Andrés Bello 2005.

Montalvo Calvimontes, Elena: Breve panorama histórico y sistemas actualizados de la catalogación en la biblioteca nacional de Bolivia (con sede en la ciudad de Sucre). http://www. bnm.me.gov.ar/novedades $/$ ? $=9350$ (8.12.2018).

Morales Bellido, Hugo: El Colegio de Profesionales en Ciencias de la Información de Bolivia CPCIB. Cinco años de labor (2005-2010). In: Fuentes, Revista de la Biblioteca y Archivo Histórico de la Asamblea Legislativa Plurinacional 4 (2010) H. 9. S. 45-53. http://www. revistasbolivianas.org.bo/scielo.php?script=sci_arttext\&pid=S199744852010000400009\&lng=es\&nrm=iso (8.12.2018).

Múnera-Torres, María-Teresa: Perfiles de formación bibliotecológica en algunas universidades de América del Sur. In: Ibersid 6 (2012). S. 95-100. http://ibersid.eu/ojs/index.php/ ibersid/article/view/4036, (8.12.2018).

Nilges, Annemarie: Nationalbibliographien Lateinamerikas. Köln: Greven 1983 (Kölner Arbeiten zum Bibliotheks- und Dokumentationswesen Heft 4). 
Oporto Ordóñez, Luis: Bibliotecas en Bolivia. In: Librínsula 4 (2007) H. 184. http://librinsula. bnjm.cu/1-205/2007/julio/184/noticias/noti1977.htm (8.12.2018).

Oporto Ordóñez, Luis: La Biblioteca del Congreso en su primer centenario. In: Fuentes, Revista de la Biblioteca y Archivo Histórico de la Asamblea Legislativa Plurinacional 5 (2011) H. 16. http://www.revistasbolivianas.org.bo/scielo.php?script=sci_arttext\&pid=S199744852011000500003\&lng=es\&nrm=iso (8.12.2018).

Oporto Ordóñez, Luis: Historia de las bibliotecas en Bolivia. In: Fuentes, Revista de la Biblioteca y Archivo Histórico de la Asamblea Legislativa Plurinacional 7 (2013) H. 29. http:// www.revistasbolivianas.org.bo/scielo.php?pid=S1997-44852013000600004\&script=sci_arttext (8.12.2018).

Oro, Nelson: Biblioteca Virtual en Salud Pública (bvsp) y temáticas Bolivia. https://www.paho. org/bol/index.php?option=com_content\&view=article\&id=522:biblioteca-virtual-saludpublica-bvsp-tematicas-bolivia\&Itemid=481 (8.12.2018).

Otero, Pedro Manuel: Bolivia: celebran campaña en favor de las bibliotecas comunitarias. In: Radio Habana Cuba (2014). http://www.la-razon.com/sociedad/Presentan-Bolivia-Leepretende-recolectar_0_2705729447.html (8.12.2018).

Pacheco Balanza, Máximo: Persönliche Mitteilung 2017 (17.5.2017). Es handelt sich um eine EMail an mich

Pampuch, Thomas u. Agustín Echalar Ascarrunz: Bolivien. 4. Aufl. München: Beck 2009 (Beck'sche Reihe 813: Länder).

Payer, Margarete: Zum Informationswesen Boliviens. http://www.payer.de/bolivien3/ bolivien03.htm (8.12.2018).

Poco desarrollo de Bibliotecas Virtuales en América Latina. In: La Jornada (24.7.2006). https://www.jornada.com.mx/2006/07/24/index.php?section=cultura\&article=a02n1cul (8.12.2018).

REDETBO. http://cepaoruro.org/?option=com_content\&view=article\&id=392:redetbo\& catid=24:redetbo\&ltemid=7 (8.12.2018).

Rico, Ramiro: Persönliche Mitteilung 2017 (23.5.2017). E-Mail an mich

Rossells, Beatriz, Luis Oporto u. Virginia Ayllón: Un país desinformado? Estudios sobre información científica y cultural en Bolivia (1993-2003). La Paz, Bolivia: Programa de Investigación Estratégica en Bolivia; UNESCO; Sol de Intercomunicación 2004.

Rossells Montalvo, Beatriz: Bibliotecas y educación en Bolivia. La Paz: Centro de Estudios Sociales 1989.

Saavedra, Jhasmany: BiblioWorks: Advocating for the Creation of Libraries for Children in Chuquisaca, Bolivia. https://digitalcollections.sit.edu/capstones/2871/ (8.12.2018)

Sistema Bibliotecario - Facultad de Tecnología. http://biblio.fcet.uagrm.edu.bo/index.aspx? AspxAutoDetectCookieSupport=1, (8.12.2018).

Smet, Egbert de: The CDS/ISIS software as an omnipresent tool for Latin American libraries and documentation centres. In: Inasp Newsletter 32 (2006). S. 4-5, (28.7.2016).

Tres de cada 100 bolivianos lee dos libros anualmente. In: Página siete - Diario nacional independiente. (09.05.2014).https://www.paginasiete.bo/sociedad/2017/5/9/tres-cada-bolivianos-libros-anualmente-137018.html (8.12.2018).

UNESCO: Global Open Access Portal. http://www.unesco.org/new/en/communication-and-information/portals-and-platforms/goap/key-organizations/latin-america-and-thecaribbean/biblioteca-digital-andina/ (8.12.2018).

Universidad Mayor de San Andrés: Bibliotecología: Información. http://bibliotecologia.umsa. bo/oferta-academica (8.12.2018). 
van den Berg, Hans: Biblioteca Etnológica Boliviana : 10 años. Cochabamba: Universidad Católica Boliviana 1991.

Vernon Jackson, William: Bolivia, Libraries in. In: Encyclopedia of library and information science. Hrsg. von Allen Kent u. Harold Lancour. 2 (1969). New York, London: Marcel Dekker 1968-2003. S. 655-659.

Zapana Calderón, Hilda u. Salomón Monasterios Calzada: Sistema de gestión de la información para la Red Municipal de Bibliotecas de El Alto. In: Revista de Bibliotecología y Ciencias de la Información 10 (2006) H. 15. S. 21-26. http://www.revistasbolivianas.org. bo/scielo.php?script=sci_arttext\&pid=S2078-533X2006000100003\&lng=es\&nrm=iso (8.12.2018). [

Zelaya, Teresa u. Marinella Salinas: Situación actual de las unidades de información en Bolivia: bibliotecas y centros de documentación. In: Revista de Bibliotecología y Ciencias de la Información 5 (2000) 6-7. S. 70-85). 